\title{
NSAID Enteropathy: A Review of the Disease Entity and Its Distinction from Crohn's Enteropathy
}

\author{
Smita Esther Raju ${ }^{1}$ Rajvinder Singh ${ }^{2}$ Mahima Raju ${ }^{3}$ \\ ${ }^{1}$ Department of Radiology, Royal Adelaide Hospital, Adelaide, \\ Australia \\ 2Department of Gastroenterology, Lyell McEwin Hospital, South \\ Australia \\ ${ }^{3}$ School of Medicine, University of Adelaide, North Terrace, \\ Adelaide, South Australia \\ J Gastrointestinal Abdominal Radiol ISGAR 2019;2:78-86
}

\begin{abstract}
Address for correspondence Smita Esther Raju, MBBS, DMRD, MD, FRANZCR, Department of Radiology, Royal Adelaide Hospital, Port Road, Adelaide 5000, Australia (e-mail: dsraju74@yahoo.com; smita.raju@sa.gov.au).
\end{abstract}
Abstract
Keywords
- computed tomogra- phy enterography
- Crohn's disease
- diaphragm disease
- enteropathy
- enteroscopy
- magnetic resonance enterography
- nonsteroidal anti-in- flammatory drugs
- small intestine
- stricture

Nonsteroidal anti-inflammatory drug (NSAID)-induced enteropathy is an increasingly recognized entity. Patients of older age and those suffering from conditions such as arthritis requiring long term NSAIDs are thought to be at greater risk. Introduction of enteroscopic techniques has greatly improved understanding of NSAID-related small intestinal injury. Complementary high-resolution cross-sectional imaging techniques aid in initial evaluation and for exclusion of alternative etiology. Erosions, superficial ulcerations, and short segment strictures are the most commonly described findings. The diagnosis of the condition lies in obtaining relevant history in addition to a high degree of suspicion during investigation of anemia, obscure gastrointestinal bleeding, small bowel obstruction, and protein losing enteropathy. Herein, the authors present a review of pathogenesis and imaging findings of NSAID enteropathy with particular emphasis on distinction from Crohn's enteropathy.

\section{Introduction}

Nonsteroidal anti-inflammatory drugs (NSAIDs) are one of the most widely prescribed medications. More than 100 million prescriptions are written in United States annually and approximately 50 billion aspirin tablets are consumed worldwide. The gastrointestinal (GI) tract appears to be the most commonly affected organ by chronic long-term use. ${ }^{1}$ The worldwide prevalence of NSAID-associated gastric or duodenal ulcers ranges from 9 to 22\%, with severe hemorrhage and obstruction occurring in less than $1 \%$ of patients. It is thought that use of enteric coated, sustained release or slow release NSAIDs may have shifted the damage from more proximal areas of the GI tract towards the distal small intestine and even the colon. Lanas et al reported this shift as a ratio of gastroduodenal to distal involvement that changed from 4:1 in 1996 to $1: 4$ in $2005 .^{2}$ In an autopsy series by Allison et al, small intestinal erosions were found in $8.4 \%$ of NSAID users compared to

received

August 29, 2018

accepted after revision

March 1, 2019

published online

July 17, 2019
$0.6 \%$ of nonusers. There were three deaths among NSAID users secondary to perforated intestinal ulcers. ${ }^{3}$ In a multicenter study, 51\% of long-term NSAID users were found to have signs of intestinal injury on double-balloon endoscopy. ${ }^{4}$ It is thought that 40 to $70 \%$ of long-term NSAID users may develop some form of enteropathy, although the incidence of diaphragm disease is only $2 \%{ }^{5}$ Diaphragm disease refers to characteristic short circumferential strictures resembling a diaphragm, which occur as a consequence of submucosal fibrosis. ${ }^{6}$ This low incidence could be due to the fact that many of the cases with diaphragm disease remain subclinical. Distinction from other enteropathies, in particular Crohn's disease, is imperative given the implications on management.

\section{Pathogenesis}

NSAID-induced intestinal injury is thought to be multifactorial in nature with increased intestinal permeability being
(C)2019 Indian Society of Gastrointestinal and Abdominal Radiology

\section{License terms}

10.1055/s-0039-1691846

ISSN 2581-9933. 
the key phenomenon unlike the stomach, where inhibition of cyclo-oxygenase activity and suppression of prostaglandin synthesis plays the key role. Bjarnason et al in an effort to explain this further proposed a "three hit" hypothesis. First, the lipids of phospholipids on the mucosal surface are solubilized, causing direct damage of epithelial mitochondria. This mitochondrial damage depletes intercellular energy leading to calcium efflux and induction of free radicles. Disruption of intercellular junction occurs subsequently leading to an increase in intestinal permeability. The mucosal barrier then becomes weak causing increased infiltration of endoluminal bile acid, bacteria, proteolytic enzymes, and toxins. ${ }^{7}$ Neutrophils and many other inflammatory mediators are therefore activated and inflammatory damage to the lining of the GI tract, such as erosions, ulcerations, bleeding, and loss of protein occurs. Additionally, enterohepatic circulation of NSAIDs and bile augments the damage to the small bowel. ${ }^{8}$

\section{Gross and Histopathological Findings}

First described by Lang et al, diaphragm disease was earlier thought to be pathognomonic of NSAIDs-related damage. ${ }^{9}$ The study identified lesions centered on pathological accentuation of plicae circularis that resulted in fixed thin septae-like projections narrowing the ileal or mid intestinal lumen to a few millimeters. The overlying mucosal changes are often minor with superficial erosions or mild villous blunting. The thickness of the septae is dependent on the degree of submucosal fibromuscular obliteration. When minimal, these are thin and often radiologically occult. When more extensive, they can achieve a humplike profile. The more affected segments could show short strictures with active mucosal ulceration. Serosal retraction is noted at focal short segment strictures causing compartmentalization of bowel lumen. ${ }^{10}$

The diaphragms are thought to reflect chronic injury and attempts at repair. In a review of 10 cases, authors described multiple diaphragms ranging from 2 to 22 in number, all with stenosis of the lumen, ring-like serosal retraction, and ulceration on the diaphragm. Histopathological features confirmed focal injury with mild increase in lymphocytes and plasma cells. Unlike normal plicae, histology revealed circumscribed focus of fibrosis at the apex of the septum in the submucosa, abutting or extending into muscularis. Neutrophilic infiltrate in lamina propria, cryptitis/crypt abscess, features of chronic injury such as architectural distortion, crypt short fall, ulcer associated cell lineage may occur. Neurovascular/muscular hamartoma-like change was noted in 9 out of 10 cases. ${ }^{11}$ Neuromuscular and vascular hamartoma was originally described as fascicles of smooth muscle derived from the muscular mucosae, bundles of unmyelinated nerve fibers with scattered ganglion cells, and angiomatous vessels, causing stenosis. ${ }^{12}$

\section{Diagnosis}

Hayashi et al defined the criteria for NSAID enteropathy as:

1. History of NSAID use
2. Endoscopic findings, such as erosions, ulcers, and diaphragm-like strictures

3. Improvement in clinical manifestations and/or endoscopic findings after stopping the NSAIDS; and

4. Exclusion of other etiologies, such as inflammatory bowel disease (IBD), infection, and malignancy. ${ }^{13}$

The sign and symptoms of NSAID-induced enteropathy are nonspecific. Indigestion, abdominal pain, constipation, diarrhea, iron deficiency anemia, and protein loss have been described. Severe life-threatening complications of bleeding, obstruction, or perforation are infrequent. ${ }^{14}$ In a systematic review, only 55 cases with NSAID-related small bowel disease required surgery over a period of 30 years. ${ }^{15}$

In the past, the diagnosis had been challenging and hence the clinical importance of the entity underestimated. It relied on measurement of markers of small bowel permeability and inflammation. Chromium ${ }^{17}$-labeled ethylenediaminetetraacetic acid was used as a marker of permeability. This is rarely absorbed by intact small bowel; however, when the small bowel is damaged, there is absorption and subsequent excretion of the product in the urine. ${ }^{16,17}$ Calprotectin is a protein in the cytosol of neutrophils, monocytes, and macrophages. It can be used as an inflammatory marker of small intestine. Unfortunately, it has low specificity and can be elevated in other inflammatory conditions especially Crohn's disease. ${ }^{18}$ The inflammation of the small intestine can also be detected by scintigraphy using 111 indium (In)labeled leukocytes. Fifty to seventy percent of patients taking long-term NSAIDs showed enhanced uptake in the small bowel, but this test was difficult to use in clinical practice due to its high cost. ${ }^{19}$

\section{Endoscopic Studies}

Video capsule endoscopy (VCE) and balloon-assisted enteroscopy (BAE) have enabled direct visualization, localization and hence better understanding of intestinal injury. Small bowel enteroscopy (SBE) is a safe and effective technique for diagnosing small bowel diseases. SBE with prior VCE seemed to improve the diagnostic yield of small bowel diseases. ${ }^{20}$ Macroscopic small bowel injury has been widely observed even after short-term NSAID use, however, the degree of involvement worsens with longer use.

A study utilized VCE to quantitate and assess the nature of the small bowel damage caused by NSAIDs when taken on a short-term basis. After 2 weeks of drug intake, 30 subjects (75\%) had increased repeat fecal calprotectin concentrations above the upper limit of normal. VCE showed new pathology in 27 subjects (68\%). The most common lesions were mucosal breaks seen in 16 subjects (40\%) (-Table 1 ). ${ }^{21}$

Comparing this with a prospective analysis of VCE findings of 143 patients with either rheumatoid or osteoarthritis who were on more than 1 month of NSAIDs; small intestinal lesions were identified in $44.8 \%$ of patients. Findings were classified into mild (multiple red spots or $<10$ erosions and/ or aphthous lesions), moderate (10-20 erosions or aphthous lesions), and severe ( $>20$ erosions or aphthous lesions, ulcers, stenosis, and/or bleeding). The lesions were mostly mild 
Table 1 Classification of endoscopic findings in NSAID injury as per Maiden et al $^{21}$

\begin{tabular}{|l|l|l|}
\hline $\begin{array}{l}\text { Classification of } \\
\text { lesions }\end{array}$ & Nature & Number/\% \\
\hline Category 1 & Reddened folds & $14 / 35$ \\
\hline Category 2 & $\begin{array}{l}\text { Denuded areas } \\
\text { with loss of villous } \\
\text { architecture }\end{array}$ & $8 / 20$ \\
\hline Category 3 & Petechiae & $13 / 33$ \\
\hline Category 4 & Mucosal breaks & $16 / 40$ \\
\hline Category 5 & $\begin{array}{l}\text { Presence of blood, no } \\
\text { lesion visualized }\end{array}$ & \\
\hline Category 6 & $\begin{array}{l}\text { Other findings- } \\
\text { angiodysplasia, } \\
\text { lymphangiectasia }\end{array}$ & \\
\hline
\end{tabular}

Abbreviation: NSAID, nonsteroidal anti-inflammatory drug.

(36.4\%), less frequently moderate $(3.5 \%)$, or severe $(4.9 \%)$. $^{22}$ VCE showed a high $(\sim 70 \%)$ rate of small bowel abnormality in chronic NSAID users ( $>3$ months); $50 \%$ patients had mild and $25 \%$ of the patients. ${ }^{23}$

While VCE is highly sensitive for mucosal lesions, the relevance of these findings is difficult to establish. In a study by Goldstein et al, mucosal erosions and petechiae were found in $\sim 14 \%$ of healthy individuals. ${ }^{24}$ Some of the limitations of VCE include inaccurate localization of the abnormalities, rapid capsule transit which can sometimes "miss" lesions, presence of small bowel fluid or debris compounding visualization, and the lack of optimal distension. ${ }^{25}$

Capsule retention is defined as lack of elimination of the camera from the digestive tract within 14 days after digestion. In a retrospective 3-year study, where radiologic workup had been negative, seven patients with NSAID enteropathy had retention of the capsule necessitating operative extraction. On average, nine diaphragms were identified in each patient by means of intraoperative palpation and enteroscopy, and confirmed at pathologic examination. ${ }^{26}$

\section{Radiological Studies}

The need for complementary imaging techniques arises due to manifold reasons. Endoscopic studies undoubtedly score high in demonstration of mucosal abnormality; however, the accuracy of mural, perimural, and extraenteric manifestation is superior with cross-sectional techniques. ${ }^{27}$ Barium or cross-sectional studies could be used as a roadmap to exclude high-grade stricturing lesions which would preclude capsule endoscopic assessment.

\section{Barium Meal Follow Through/Enteroclysis}

Ring or diaphragm like as well as broad based strictures due to NSAID injury have been described in a long segment of jejunum. Compression spot views showed diaphragms to be thicker than adjacent folds. Broader strictures with humps were seen as producing a lifesaver-like or bagel-like configuration. ${ }^{28}$ The strictures are usually found close to each other in a segment of small bowel either on imaging or on subsequent laparotomy. ${ }^{29}$ Levi et al also described diaphragm-like strictures secondary to prolonged NSAID use in a case series of four patients. ${ }^{30}$

The advantage of barium studies would be distinction of a fixed stricture from a transient abnormality due to peristalsis. Limitations include dependence on operator ability and overlap of loops obscuring some information. Patient compliance is another factor affecting use of enteroclysis techniques.

Barium studies are not advocated routinely in detection of NSAID-related intestinal injury. These, however, have been able to depict circular narrowing as pseudo folds in many cases. This sign could predict capsular retention and can be utilized as a patency tool. ${ }^{31}$

\section{CT and MR Enterography/Enteroclysis}

Computed tomography (CT) and magnetic resonance imaging (MRI) are the current standard for small bowel assessment as per ECCO, the European Crohn's and Colitis Organisation. Both require luminal distension for adequate visualization of the small bowel, as well as intravenous contrast. Luminal distension can be achieved through orally administered contrast (enterography) or through a nasojejunal tube (enteroclysis). Diagnostic accuracy in CT and MRI enterography is generally the same, with slightly higher specificity for enteroclysis. Enterography is preferred due to ease of administration, better patient tolerance, and lesser radiation dose in case of CT. Jejunal distension may not be adequate and enteroclysis may provide additional benefit in case of strong suspicion of jejunal involvement. ${ }^{32-34}$

A high degree of suspicion is required as the thin diaphragms could be radiologically occult as in these cases, the outer contour of bowel could be maintained. Multiple, short-segment strictures are the hallmarks of imaging diagnosis. Strictures may have minimal hyperenhancement or wall thickening, but these findings are typically symmetric and circumferential with respect to the bowel lumen. ${ }^{35}$

A diaphragm-like stricture was found to be the most common finding in a retrospective analysis by Flicek et al, highlighting that the detection may be limited to the more advanced cases. ${ }^{36}$ These strictures were noted involving ileum more than the jejunum. Typically, these were multiple, circumferential, ring-like, short segment (5-10 mm length). Mild segmental mural thickening (e.g., 5-8 mm) and variable mucosal hyperenhancement were noted. The latter when present suggested active inflammation and aided in detection of strictures. Small bowel dilatation was not significant and present in less than half with maximum luminal diameter being $3.8 \mathrm{~cm}$ (-Figs. 1A, B and 2A, B).

In a review of 11 patients with drug-induced small bowel damage (8 NSAIDs and 3 aspirin), abnormal findings in the small bowel were detected by CTE (CT enterography) in $8 / 11$ patients (73\%). Multiple lesions were found in 5/8 patients (63\%). In addition, multiple lesions were shown in short-term users (6 months or shorter). The authors classified the findings and their endoscopic correlation in three subsets as depicted in - Table 2 . $^{37}$

The circumferential nature of abnormality and often the lack of significant proximal dilatation necessitate the 
Table 2 Classification of CTE findings in NSAID enteropathy by Kishi et al ${ }^{37}$

\begin{tabular}{|l|l|l|l|l|}
\hline Type & Characteristics & Findings & Incidence & Endoscopic correlation \\
\hline 1 & Mucosal & $\begin{array}{l}\text { Strong mucosal hyperenhancement, } \\
\text { no mural thickening }\end{array}$ & $\begin{array}{l}4 / 8 \text { patients, 12/22 } \\
\text { lesions, all short- } \\
\text { term users }\end{array}$ & $\begin{array}{l}\text { Small erosions, ulcers } \\
\text { with mild edema, and } \\
\text { redness }\end{array}$ \\
\hline 2 & $\begin{array}{l}\text { Homogenous } \\
\text { hyperenhancement }\end{array}$ & $\begin{array}{l}\text { Homogenous and strong mural } \\
\text { enhancement }\end{array}$ & $\begin{array}{l}2 / 8 \text { patients, 4/22 } \\
\text { lesions, all long- } \\
\text { term (more than } \\
3 \text { years) users }\end{array}$ & $\begin{array}{l}\text { Large ulcers with edema } \\
\text { and redness Perienteric } \\
\text { mesenteric stranding } \\
\text { and reactive mesenteric } \\
\text { nodes noted }\end{array}$ \\
\hline 3 & $\begin{array}{l}\text { Stratified } \\
\text { enhancement }\end{array}$ & $\begin{array}{l}\text { Stratified or target appearance with } \\
\text { marked wall thickening }\end{array}$ & $\begin{array}{l}\text { 4/8 patients, 6/22 } \\
\text { lesions, both short- } \\
\text { and long-term } \\
\text { users }\end{array}$ & $\begin{array}{l}\text { These patients had } \\
\text { luminal strictures, and } \\
\text { the lesions corresponded } \\
\text { to annular or large ulcers } \\
\text { associated with strictures } \\
\text { observed on endoscopic } \\
\text { examination }\end{array}$ \\
\hline
\end{tabular}

Abbreviations: CTE, computed tomography enterography; NSAID, nonsteroidal anti-inflammatory drug.
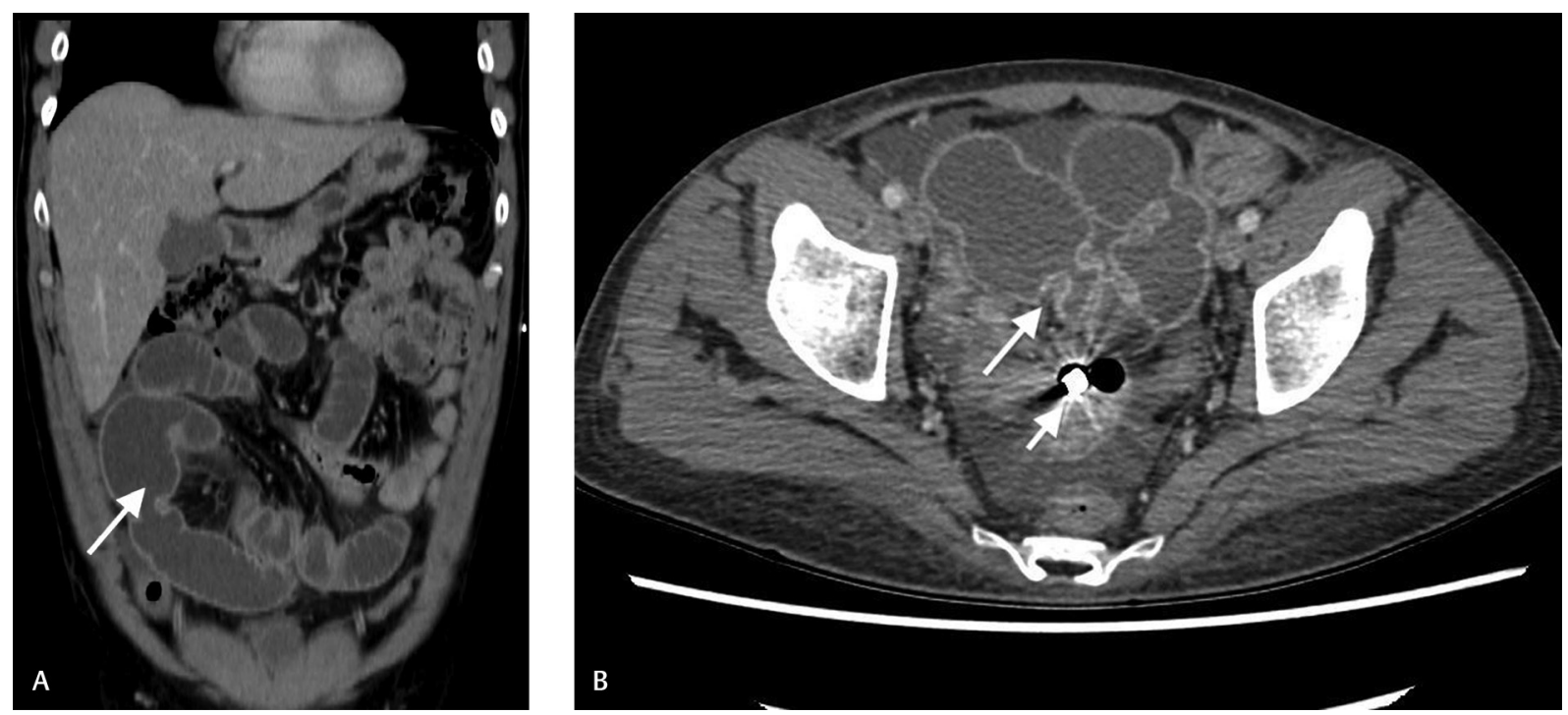

Fig. 1 (A) A 60-year-old man presenting with intermittent abdominal pain of a few months' duration. Computed tomography (CT) portal venous phase coronal reconstruction showing a symmetric short segment diaphragm like stricture (arrow) in a distal ileal loop. (B) A 33-yearold male under investigation for suspected protein losing enteropathy. Known background of nonsteroidal anti-inflammatory drug abuse due to previous (motor vehicle) accident and chronic backache. CT portal venous phase axial image showing a short segment symmetric stricture in distal ileum (arrow). Retained capsule also noted in an adjacent segment (short arrow). Ascites and mild abdominal wall edema reflecting hypoproteinemia.

careful orthogonal inspection of bowel and imaging in multiple phases to confirm the presence of stricture. An elaborate discussion on optimal MRE (MR enterography) technique for small bowel imaging is beyond the scope of current review. Reduction in peristalsis/motion artefacts by administration of antiperistaltic agents and using faster acquisition techniques such as half-Fourier rapid acquisition with relaxation enhancement or single shot fast spin-echo which allow each image to be obtained in less than 1 second is of paramount importance in small bowel imaging. Balanced gradient echo sequences are useful in providing mural and extramural information. Dynamic contrast-enhanced fat saturated T1-weighted sequences aid in detection of mural inflammation, small bowel and mesenteric vasculature, and in excluding malignant causes of small bowel obstruction. ${ }^{38}$
The literature on MRE assessment of diaphragm disease is limited. Frye et al highlighted the advantage of different pulse sequences employed in MRE and enteroclysis offering multiple time points over which a stricture can be visualized, increasing radiologist confidence. Contrast-enhanced sequences are helpful as the diaphragms enhance differently from adjacent small bowel folds ( - Figs. 3A, B and 4). There is an added advantage of lack of ionizing radiation when imaging younger patients.

Extramural findings are not a feature of diaphragm disease. A single case report described small bowel diaphragm disease mimicking a malignancy such as lymphoma due to multiple ileal strictures and florid mesenteric lymphadenopathy. Histopathology showed evidence of diaphragm disease and reactive nodes. ${ }^{39}$ Two of eight patients studied by Kishi et al showed mesenteric stranding accompanying mural thickening. ${ }^{37}$ 

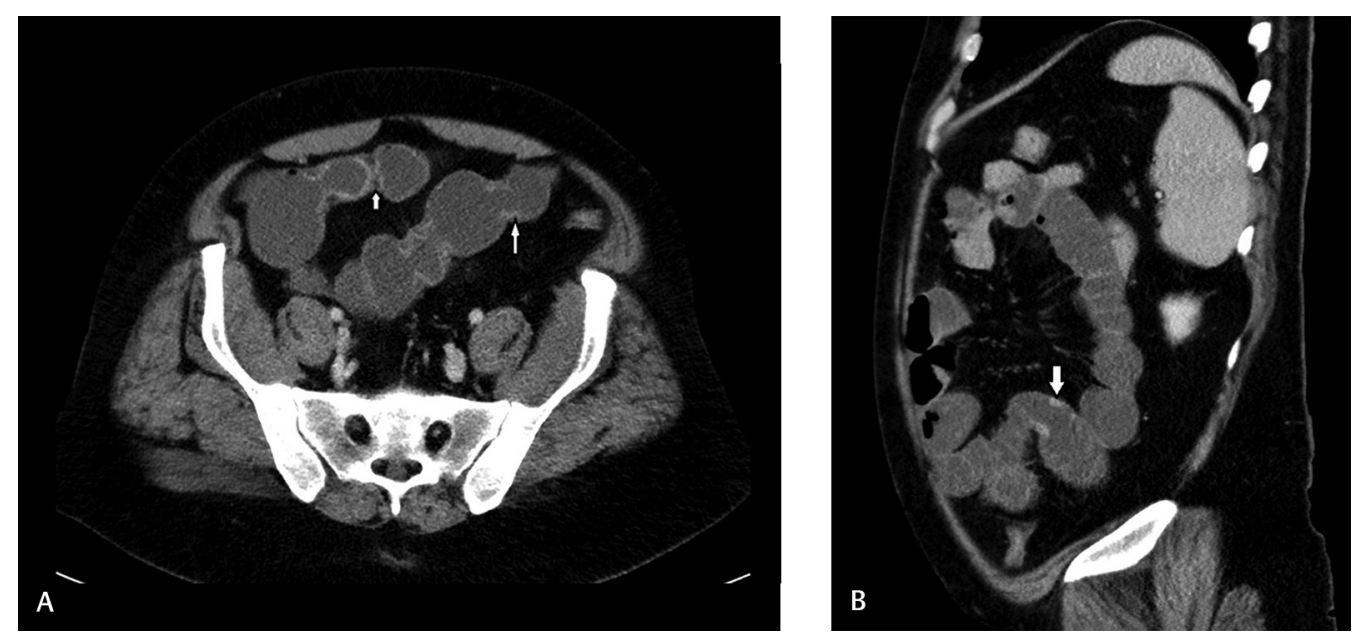

Fig. 2 A 45-year-old male with background of chronic nonsteroidal anti-inflammatory drug abuse presented with acute abdominal pain and distension. (A) An axial computed tomography enterography (CTE) post-contrast image showing multiple diaphragm like strictures (two of these are indicated by white arrows) in ileal loops; some of these strictures appear as accentuated folds with submucosal fat infiltration. (B) CTE sagittal image showing short segment symmetric mural thickening and enhancement (white arrow). No overt mesenteric changes demonstrated.
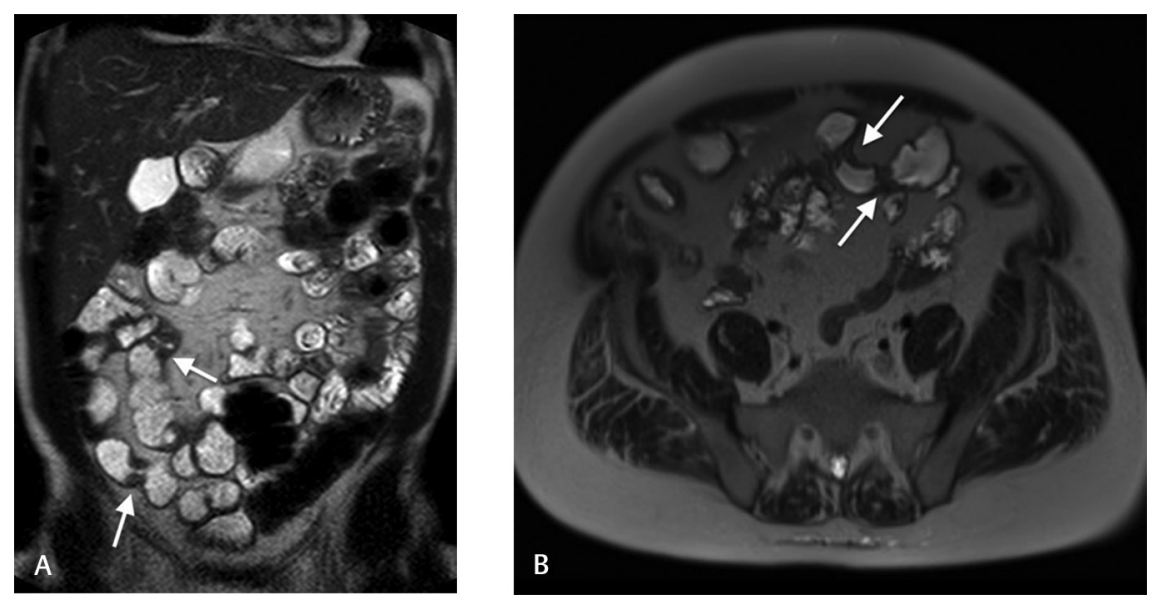

Fig. 3 A 45-year-old male with background of chronic nonsteroidal anti-inflammatory drug abuse presented with acute abdominal pain and distension magnetic resonance enterography T2 Haste multi-breath hold coronal (A) and axial (B) images showing short segment symmetric strictures (arrows) in mid and distal ileal loops.

\section{Differential Diagnosis}

Once thought pathognomonic, diaphragm disease is not exclusive to NSAID-induced enteropathy. Diaphragm-like strictures have been described in patients with no documented NSAID use. In a study by Wang et al, five patients with diaphragm-like strictures were described all localized in the mid-to-distal ileum with no extramural or mesenteric findings. Unlike NSAID enteropathy, these were isolated findings with no evidence of erosions, ulceration, inflammation elsewhere in the bowel. Apart from one case where two strictures were identified, all other cases showed a solitary stricture, again contrasting with NSAID-related diaphragm disease where multiplicity is a common feature. ${ }^{40,41}$ Congenital diaphragm-like strictures or webs presenting in adulthood are a well-known entity. ${ }^{42,43,44}$ Cytomegalovirus infection and eosinophilic enteritis have been described to have diaphragm-like strictures. ${ }^{45,46}$ Multifocal stenosing ulcerations are known in Crohn's, vasculitis, radiation enteritis, and cryptogenic multifocal ulcerous stenosing enteritis (CMUSE) among other conditions. ${ }^{47}$ CMUSE is defined as unexplained small intestinal multiple strictures and ulcerations of unknown origin and is thought to be an atypical form of vasculitis. It is seen in middle-aged patients with no evidence of systemic inflammation. Symptoms can show improvement with steroids and can lead to dependence. It can relapse chronically or after surgery. ${ }^{48}$

\section{NSAID versus Crohn's Disease}

The distinction from adult onset small bowel stricturing phenotype of Crohn's enteropathy is of prime consideration. Crohn's disease is an idiopathic inflammatory disorder of unknown etiology with genetic, immunologic, and environmental influences, characterized by a chronic progressive destructive course in the small or large bowel..$^{49}$ There are truly no pathognomonic features. Diagnosis of Crohn's disease is based on a combination of clinical presentation and endoscopic, radiologic, histologic, and pathologic findings. 


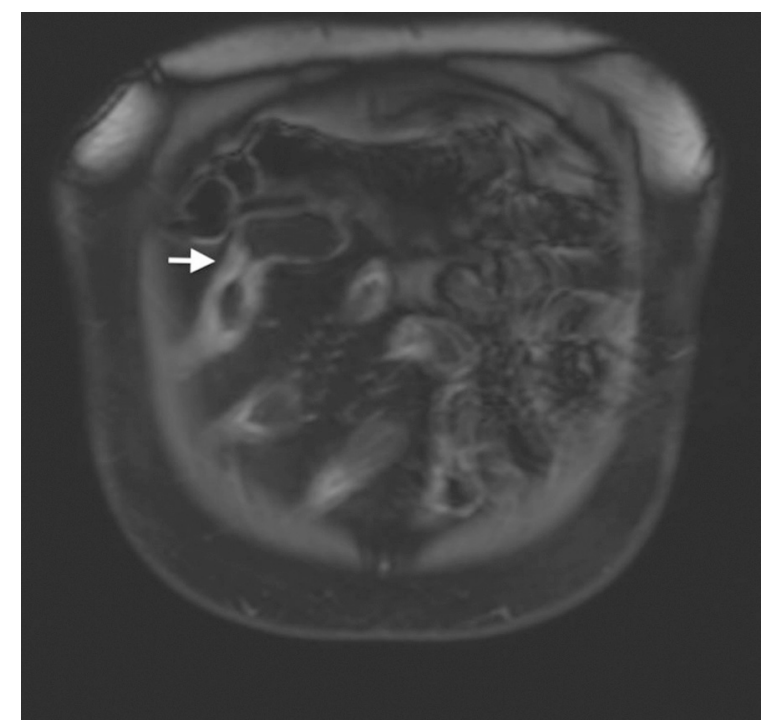

Fig. 4 Magnetic resonance enterography T1 vibe fat saturated post-gadolinium axial image showing an ileal stricture (arrow) with mild symmetric mural thickening and hyperenhancement. No significant perienteric inflammatory change.

Classical granulomatous inflammation is seen in a minority of patients (up to 33\%) with Crohn's disease, and is helpful, but not required, for diagnosis. ${ }^{50}$

While abdominal pain, fatigue secondary to anemia, can be seen in NSAID enteropathy, diarrhea is not a common feature. Other hallmark/cardinal symptoms of Crohn's disease include weight loss, fever, growth failure, and recurrent fistulas. Extraintestinal manifestations can also be a presenting feature. ${ }^{51}$ It has an annual incidence ranging from 3 to 20 cases per $100,000 .{ }^{17}$ The median age of onset is much younger at 30 years. It has two peaks, the first between age 20 and 30 years and a smaller peak in older patients above 50 .

Colonoscopy with intubation of the terminal ileum and biopsy is recommended as part of the initial evaluation of patients with suspected IBD. Over $80 \%$ of patients with IBD will have mucosal involvement within the reach of the colonoscope. Ileal intubation rates via colonoscopy are as high as 80 to $97 \%$ in patients in whom the cecum is reached. ${ }^{52} \mathrm{CE}$ or BAE, small bowel barium studies, and cross-sectional imaging help evaluate the small bowel disease when isolated.

Differences between NSAID and Crohn's enteropathy were reported in a study of 1,644 symptomatic patients by single-balloon enteroscopy over a period of 10 years. Erosion and superficial ulcers were equally evident in NSAIDs injury, whereas deep ulceration was a common finding in Crohn's disease. Near equal distribution of lesions in the jejunum and ileum occurred in NSAID enteropathy, while there was a strong (13:1) predilection for ileal disease in Crohn's disease. Ileocecal involvement was $21 \%$ with NSAIDs and $36 \%$ in Crohn's disease. No specific relation of ulcers to mesentery was seen in NSAID enteropathy. In Crohn's disease, ulcers were longitudinal involving the mesenteric border. Short diaphragm-like strictures were typical of NSAIDs injury, whereas eccentric longer segment involvement was commonly seen in Crohn's disease. ${ }^{53}$ In a review of SBE findings in Crohn's disease, other than the typical longitudinal ulceration at the mesenteric border, there were findings of cobble stoning, fistulae (usually proximal to stricture), and pseudodiverticulae; none of which have been described in NSAID injury. In addition, adenocarcinoma or lymphoma may complicate Crohn's disease. ${ }^{54}$

Small bowel imaging should be performed as part of initial evaluation for Crohn's disease. CTE has a sensitivity of $90 \%$ for the detection of small bowel disease in patients with Crohn's disease and is comparable to magnetic resonance enterography (MRE).$^{55}$ Because of the absence of any radiation exposure, MRE should be used preferentially in young patients ( $<35$ years) and in patients in whom it is likely that serial exams will need to be performed. ${ }^{56}$

The findings of Crohn's disease on CTE/MRE can be categorized into four broad subsets ${ }^{57}$ inflammation, fistula, abscess, and stenosis. Penetrating disease with fistulization or abscess formation is a consequence of transmural inflammation and deep ulceration, whereas in NSAID enteropathy, ulceration is often superficial and the serosa intact.

Evidence of active inflammation is in the form of mural thickening ( $>3 \mathrm{~mm}$, severe if $>6 \mathrm{~mm}$ ), hyperenhancement, ulceration/cobblestone appearance, increased mesenteric vascularity (comb's sign), regional lymphadenopathy (mesenteric lymph nodes $>10 \mathrm{~mm}$ ), and creeping fat (fibrofatty mesenteric proliferation adjacent to a chronically inflamed bowel that persists in quiescent phase $)^{59}(- \text { Fig. 5 })^{56}$. The mesentery is usually normal or shows minimal inflammation in NSAID enteropathy. Multifocal symmetric mural thickening and mild hyperenhancement are noted often of a lesser degree than with Crohn's disease.

Stenosing disease could be secondary to active inflammation with edema and spasmodic contraction or secondary to transmural fibrosis. The latter is not associated with $\mathrm{T} 2$ hyperintense submucosal edema or features of mesenteric inflammation in contrast to the short segment or web-like symmetric strictures of NSAID injury. Long-segment $(2-3 \mathrm{~cm}$ to $30 \mathrm{~cm}$ ) involvement with terminal ileal predominance, skip lesions, asymmetric nature with preferred mesenteric border thickening, pseudosacculations of the antimesenteric border, abundant adjacent fibrofatty mesenteric proliferation with separation of bowel loops is typical of Crohn's strictures. ${ }^{56-60}$ Proximal dilatation $>3 \mathrm{~cm}$ could be present in functional Crohn's strictures ( - Figs. 6, 7A and B).

\section{Management of NSAID Enteropathy}

Cessation of NSAID use can revert some of the changes observed with chronic use. To reduce the risk of complications, such as ulcers, bleeding, and obstruction, in chronic NASID users, many researchers have attempted to treat and prevent these disorders by administration of metronidazole, sulfasalazine, cyclooxygenase 2 inhibitors, misoprostol, rebamipide, human lactoferrin, and fish protein hydrolysate. Data on long-term usage is not yet available. Careful monitoring and special attention for the indications of NSAIDs are required to avoid this disorder in individuals taking NSAIDs. ${ }^{61,62}$ 
BAE can enable dilatation of strictures, although it has to be used judiciously and with caution. ${ }^{63}$ Laparotomy and small bowel resection for obstruction secondary to small bowel diaphragms may be required. ${ }^{26}$

\section{Conclusion}

NSAID-related small bowel injury is common especially among chronic users. Endoscopic techniques are highly sensitive in demonstrating NSAID-related mucosal injury. The

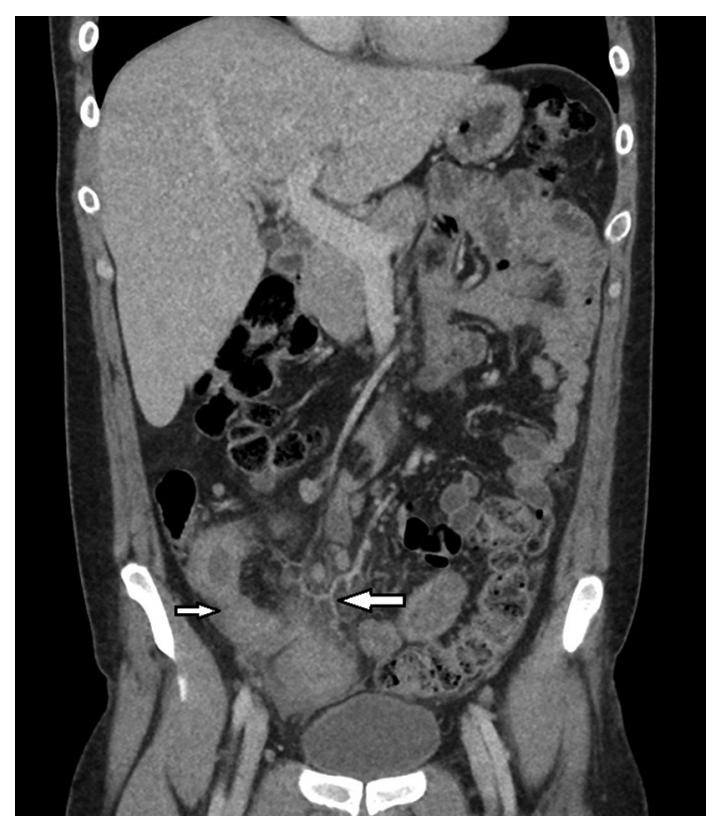

Fig. 5 Computed tomography abdomen and pelvis portal venous phase coronal reconstruction showing active Crohn's ileitis with mural thickening and hyperenhancement of a long distal ileal segment (thin arrow) associated with mesenteric stranding, hypervascularity (thick arrow), and adenopathy. relevance of subtle findings detected is debatable. Cross-sectional techniques can aid in providing a roadmap for enteroscopy and subsequent management by highlighting diaphragm-like strictures when present and excluding extramural involvement or alternative etiologies when present. CTE and MRE have shown similar sensitivity in small bowel assessment. More data is available on CTE findings of NSAID strictures possibly reflecting its availability, lower cost, and superior spatial resolution in the detection of short

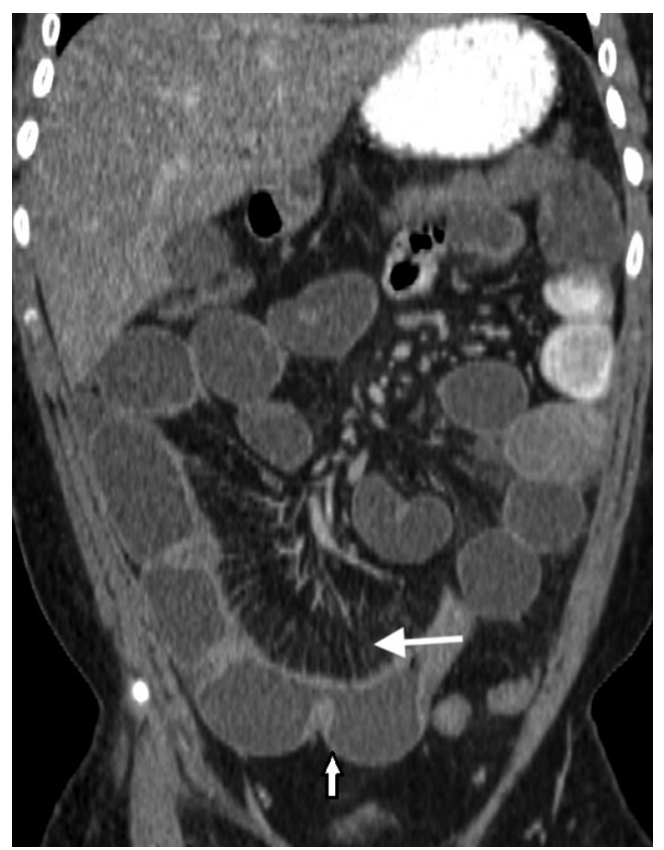

Fig. 6 Computed tomography enterography coronal post-contrast image in a patient with known background of Crohn's showing asymmetric involvement of a long ileal segment with straightening of mesenteric border and antimesenteric sacculations (small arrow). Prominent mesenteric vascularity (comb's sign) is also noted (big arrow).
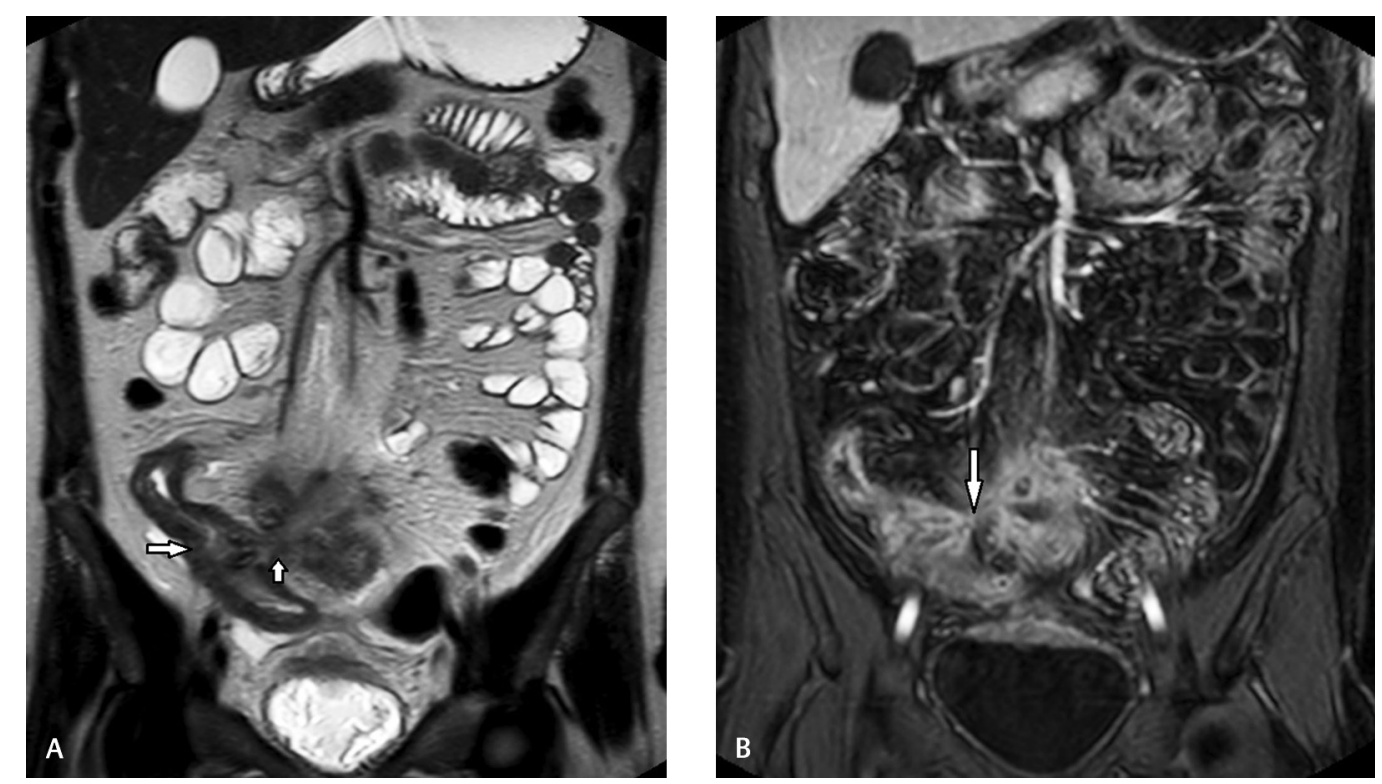

Fig. 7 (A) Magnetic resonance enterography T2 Haste coronal image in a patient with Crohn's ileitis showing a long segment structuring disease (big arrow) with evidence of penetration at the mesenteric border (small arrow). (B) Coronal T1 FS post-contrast image in a patient with Crohn's ileitis showing penetrating disease in form of interloop adhesions and entero-enteric communication (arrow). 
segment strictures. Short segment, symmetric, multifocal diaphragm-like strictures with mild mural thickening and variable hyper enhancement are typical of NSAID enteropathy in contrast to long segment, asymmetric longitudinal ulcers on the mesenteric border in Crohn's disease. Penetrating disease is absent and mesenteric findings are uncommon or mild if present in NSAID-related disease.

\section{Conflict of Interest}

None declared.

\section{References}

1 Gupta M, Eisen GM. NSAIDs and the gastrointestinal tract. Curr Gastroenterol Rep 2009;11(5):345-353

2 Lanas A, García-Rodríguez LA, Polo-Tomás M, et al. Time trends and impact of upper and lower gastrointestinal bleeding and perforation in clinical practice. Am J Gastroenterol 2009;104(7):1633-1641

3 Allison MC, Howatson AG, Torrance CJ, Lee FD, Russell RI. Gastrointestinal damage associated with the use of nonsteroidal anti-inflammatory drugs. N Engl J Med 1992;327(11):749-754

4 Matsumoto T, Kudo T, Esaki M, et al. Prevalence of non-steroidal anti-inflammatory drug-induced enteropathy determined by double-balloon endoscopy: a Japanese multicenter study. Scand J Gastroenterol 2008;43(4):490-496

5 Ishihara M, Ohmiya N, Nakamura M, et al. Risk factors of symptomatic NSAID-induced small intestinal injury and diaphragm disease. Aliment Pharmacol Ther 2014;40(5):538-547

6 De Petris G, López JI. Histopathology of diaphragm disease of the small intestine: a study of 10 cases from a single institution. Am J Clin Pathol 2008;130(4):518-525

7 Bjarnason I, Hayllar J, MacPherson AJ, Russell AS. Side effects of nonsteroidal anti-inflammatory drugs on the small and large intestine in humans. Gastroenterology 1993;104(6):1832-1847

8 Wallace JL. NSAID gastropathy and enteropathy: distinct pathogenesis likely necessitates distinct prevention strategies. Br J Pharmacol 2012;165(1):67-74

9 Lang J, Price AB, Levi AJ, Burke M, Gumpel JM, Bjarnason I. Diaphragm disease: pathology of disease of the small intestine induced by non-steroidal anti-inflammatory drugs. J Clin Pathol 1988;41(5):516-526

10 Price AB. Pathology of drug-associated gastrointestinal disease. Br J Clin Pharmacol 2003;56(5):477-482

11 De Petris G, López JI. Histopathology of diaphragm disease of the small intestine: a study of 10 cases from a single institution. Am J Clin Pathol 2008;130(4):518-525

12 de Sanctis S, Qureshi T, Stebbing JF. Clinical and pathological overlap in nonsteroidal anti-inflammatory drug-related small bowel diaphragm disease and the neuromuscular and vascular hamartoma of the small bowel. Am J Surg Pathol 2001;25(4):539-541

13 Hayashi Y, Yamamoto H, Taguchi H, et al. Nonsteroidal anti-inflammatory drug-induced small-bowel lesions identified by double-balloon endoscopy: endoscopic features of the lesions and endoscopic treatments for diaphragm disease. J Gastroenterol 2009;44(Suppl 19):57-63

14 Park SC, Chun HJ, Kang CD, Sul D. Prevention and management of non-steroidal anti-inflammatory drugs-induced small intestinal injury. World J Gastroenterol 2011;17(42):4647-4653

15 Slesser A, Wharton R, Smith GV, Buchanan GN. Systematic review of small bowel diaphragm disease requiring surgery. Colorectal Dis 2012;14(7):804-813

16 Aabakken L, Osnes M. 51Cr-ethylenediaminetetraacetic acid absorption test. Effects of naproxen, a non-steroidal, anti-inflammatory drug. Scand J Gastroenterol 1990; 25(9):917-924

17 Molodecky NA, Soon IS, Rabi DM, et al. Increasing incidence and prevalence of the inflammatory bowel diseases with time, based on systematic review. Gastroenterology 2012;142(1):46-54

18 Lanas A, Sopeña F. Nonsteroidal anti-inflammatory drugs and lower gastrointestinal complications. Gastroenterol Clin North Am 2009;38(2):333-352

19 Shin SJ, Noh C-K, Lim SG, Lee KM, Lee KJ. Non-steroidal anti-inflammatory drug-induced enteropathy. Intest Res 2017;15(4):446-455

20 Ma JJ, Wang Y, Xu XM, et al. Capsule endoscopy and single-balloon enteroscopy in small bowel diseases: competing or complementary? World J Gastroenterol 2016;22(48):10625-10630

21 Maiden L, Thjodleifsson B, Theodors A, Gonzalez J, Bjarnason I. A quantitative analysis of NSAID-induced small bowel pathology by capsule enteroscopy. Gastroenterology 2005; 128(5):1172-1178

22 Tachecí I, Bradna P, Douda T, et al. Small intestinal injury in NSAID users suffering from rheumatoid arthritis or osteoarthritis. Rheumatol Int 2016;36(11):1557-1561

23 Graham DY, Opekun AR, Willingham FF, Qureshi WA. Visible small-intestinal mucosal injury in chronic NSAID users. Clin Gastroenterol Hepatol 2005;3(1):55-59

24 Goldstein JL, Eisen GM, Lewis B, Gralnek IM, Zlotnick S, Fort JG; Investigators. Video capsule endoscopy to prospectively assess small bowel injury with celecoxib, naproxen plus omeprazole, and placebo. Clin Gastroenterol Hepatol 2005;3(2):133-141

25 Gay G, Delvaux M, Rey JF. The role of video capsule endoscopy in the diagnosis of digestive diseases: a review of current possibilities. Endoscopy 2004;36(10):913-920

26 Kelly ME, McMahon LE, Jaroszewski DE, Yousfi MM, De Petris G, Swain JM. Small-bowel diaphragm disease: seven surgical cases. Arch Surg 2005;140(12):1162-1166

27 Crook DW, Knuesel PR, Froehlich JM, et al. Comparison of magnetic resonance enterography and video capsule endoscopy in evaluating small bowel disease. Eur J Gastroenterol Hepatol 2009;21(1):54-65

28 Zalev AH, Gardiner GW, Warren RE. NSAID injury to the small intestine. Abdom Imaging 1998;23(1):40-44

29 Abrahamian GA, Polhamus CD, Muskat P, Karulf RE. Diaphragm-like strictures of the ileum associated with NSAID use: a rare complication. South Med J 1998;91(4):395-397

30 Levi S, de Lacey G, Price AB, Gumpel MJ, Levi AJ, Bjarnason I. "Diaphragm-like" strictures of the small bowel in patients treated with non-steroidal anti-inflammatory drugs. Br J Radiol 1990;63(747):186-189

31 Matsumoto T, Esaki M, Kurahara K, et al. Double-contrast barium enteroclysis as a patency tool for nonsteroidal anti-inflammatory drug-induced enteropathy. Dig Dis Sci 2011;56(11):3247-3253

32 Minordi LM, Vecchioli A, Mirk P, Bonomo L. CT enterography with polyethylene glycol solution vs CT enteroclysis in small bowel disease. Br J Radiol 2011;84(998):112-119

33 Negaard A, Paulsen V, Sandvik L, et al. A prospective randomized comparison between two MRI studies of the small bowel in Crohn's disease, the oral contrast method and MR enteroclysis. Eur Radiol 2007;17(9):2294-2301

34 Torkzad MR, Lauenstein TC. Enteroclysis versus enterography: the unsettled issue. Eur Radiol 2009;19(1):90-91

35 Frye JM, Hansel SL, Dolan SG, et al. NSAID enteropathy: appearance at CT and MR enterography in the age of multi-modality imaging and treatment. Abdom Imaging 2015;40(5):1011-1025

36 Flicek KT, Hara AK, De Petris G, Pasha SF, Yadav AD, Johnson CD. Diaphragm disease of the small bowel: a retrospective review of CT findings. AJR Am J Roentgenol 2014;202(2):W140-5 
37 Kishi T, Shimizu K, Hashimoto S, et al. CT enteroclysis/enterography findings in drug-induced small-bowel damage. $\mathrm{Br} \mathrm{J}$ Radiol 2014;87(1044):20140367

38 Masselli G, Gualdi G. MR imaging of the small bowel. Radiology 2012;264(2):333-348

39 Sarantitis I, Gerrard AD, Teasdale R, Pettit S. Small bowel diaphragm disease mimicking malignancy. BMJ Case Rep 2015;2015

40 Wang M-L, Miao F, Tang Y-H, Zhao X-S, Zhong J, Yuan F. Special diaphragm-like strictures of small bowel unrelated to non-steroidal anti-inflammatory drugs. World J Gastroenterol 2011;17(31):3596-3604

41 Santolaria S, Cabezali R, Ortego J, Castiella T, Salinas JC, Lanas A. Diaphragm disease of the small bowel: a case without apparent nonsteroidal anti-inflammatory drug use. J Clin Gastroenterol 2001;32(4):344-346

42 Smiley K, Perry M, McClelland R. Congenital duodenal diaphragm in the adult: review of the literature and report of a case. Ann Surg 1967;165(4):632-636

43 Agha FP, Jenkins JJ. Ileal mucosal diaphragm causing small bowel obstruction. Gastrointest Radiol 1983;8(1):57-59

44 Moore DJ, O'Sullivan G, Hederman WP. Congenital jejunal mucosal diaphragm and phytobezoar: a cause of intermittent small bowel obstruction in an adult. Ir J Med Sci 1981;150(5):160-161

45 Shimizu T, Marusawa H, Yamashita Y. Image of the month. Diaphragm-like stricture of the small intestine related to cytomegalovirus infection. Clin Gastroenterol Hepatol 2010;8(9):A21

46 Pasha SF, Leighton JA, Williams JW, De Petris G, Harold K, Shiff AA. Capsule retention in a patient with eosinophilic gastroenteritis mimicking diaphragm disease of the small bowel. Endoscopy 2009;41(41, Suppl 2):E290-E291

47 Hwang J, Kim JS, Kim AY, et al. Cryptogenic multifocal ulcerous stenosing enteritis: radiologic features and clinical behavior. World J Gastroenterol 2017;23(25):4615-4623

48 Chung SH, Jo Y, Ryu SR, et al. Diaphragm disease compared with cryptogenic multifocal ulcerous stenosing enteritis. World J Gastroenterol 2011;17(23):2873-2876

49 Lichtenstein GR, Loftus EV, Isaacs KL, Regueiro MD, Gerson LB, Sands BE. ACG clinical guideline: management of Crohn's disease in adults. Am J Gastroenterol 2018;113(4):481-517

50 Reinisch S, Schweiger K, Pablik E, et al. An index with improved diagnostic accuracy for the diagnosis of Crohn's disease derived from the Lennard-Jones criteria. Aliment Pharmacol Ther 2016;44(6):601-611

51 Mekhjian HS, Switz DM, Melnyk CS, Rankin GB, Brooks RK. Clinical features and natural history of Crohn's disease. Gastroenterology 1979;77(4 Pt 2) :898-906

52 Coremans G, Rutgeerts P, Geboes K, Van den Oord J, Ponette E, Vantrappen G. The value of ileoscopy with biopsy in the diagnosis of intestinal Crohn's disease. Gastrointest Endosc 1984;30(3):167-172

53 Moreels TG. Small bowel enteroscopy in Crohn's disease. Ann Gastroenterol 2012;25(1):14-20

54 Xu N, Yu Z, Cao X, Wang Z, Yan M. Characteristics of nonsteroidal anti-inflammatory drugs (NSAIDs)-induced small bowel injury identified by single-balloon endoscopy or capsule endoscopy. Med Sci Monit 2017;23:5237-5245

55 Siddiki HA, Fidler JL, Fletcher JG, et al. Prospective comparison of state-of-the-art MR enterography and CT enterography in small-bowel Crohn's disease. Am J Roentgenol 2009; 193(1):113-121

56 Solem CA, Loftus EV Jr, Fletcher JG, et al. Small-bowel imaging in Crohn's disease: a prospective, blinded, 4-way comparison trial. Gastrointest Endosc 2008;68(2):255-266

57 Gauci J, Sammut L, Sciberras M, et al. Small bowel imaging in Crohn's disease patients. Ann Gastroenterol 2018; 31(4):395-405

58 Fidler J. MR imaging of the small bowel. Radiol Clin North Am 2007;45(2):317-331

59 Kilcoyne A, Kaplan JL, Gee MS. Inflammatory bowel disease imaging: current practice and future directions. World J Gastroenterol 2016;22(3):917-932

60 Chang C-W, Wong J-M, Tung C-C, Shih I-L, Wang H-Y, Wei S-C. Intestinal stricture in Crohn's disease. Intest Res 2015;13(1):19-26

61 Higuchi K, Umegaki E, Watanabe T, et al. Present status and strategy of NSAIDs-induced small bowel injury. J Gastroenterol 2009;44(9):879-888

62 Park SC, Chun HJ, Kang CD, Sul D. Prevention and management of non-steroidal anti-inflammatory drugs-induced small intestinal injury. World J Gastroenterol 2011;17(42):4647-4653

63 Gill RS, Kaffes AJ. Small bowel stricture characterization and outcomes of dilatation by double-balloon enteroscopy: a single-centre experience. Therap Adv Gastroenterol 2014; 7(3):108-114 